\title{
A Map-matching Method Using a Chi-squared Statistic for Car-navigation Systems
}

\author{
Toshiyuki AOKI ${ }^{\dagger}$, Mikio BANDO ${ }^{\dagger}$, Tomoaki HIRUTA ${ }^{\dagger}$, \\ Koichi KATO ${ }^{\dagger \dagger}$, Akihiro KAWABATA ${ }^{\dagger \dagger}$, Zhixing LIU $^{\dagger \dagger}$ \\ $\dagger \quad$ Hitachi Research Laboratory, Hitachi, Ltd. \\ 1-1, Omika-cho 7-chome, Hitachi-shi, Ibaraki, 319-1292 Japan \\ $\dagger$ Core Engineering Development Department R\&D Division, Clarion Co., Ltd.
}

E-mail: toshiyuki.aoki.gh@ @itachi.com

\begin{abstract}
In car-navigation systems, car position measurement estimates the car's position and heading angle from the outputs of a GPS receiver and sensors, and calculates the position on a link that represents a road and is recorded in a digital road map. We focus on the latter process, which is map matching. If there are large errors in the car's estimated position and heading angle and in link position and direction angle, map matching selects an incorrect link. If map matching uses the data of the past route, it can select a correct link when the car is on a parallel road. This paper proposes a map-matching method using a chi-squared statistic for car-navigation systems. This method calculates a selection criterion that takes into account the errors in the car's estimated position and heading angle and in the link position and direction angle. This criterion is chi-squared distributed and is calculated from data points that were observed at constant distance intervals. The method's performance was experimentally evaluated using observation data collected in a car. It was found to be effective on roads that pose map matching difficulties, i.e., forks in roads, parallel roads, and roundabouts.
\end{abstract}

\section{Introduction}

This paper proposes a map-matching method using the chi-squared statistic for car-navigation systems. In regard to car-navigation systems, car position measurement involves two processes: position estimation and map matching. The former process estimates the position, speed, and heading angle, and their estimation-error covariance from the output of a GPS receiver and sensors [1], [2]. The latter process selects the links near the car's estimated position, calculates the position and heading angle of candidate points on the links (link candidates) and selects the best link candidate, as shown in Fig. 1. Here, links represent roads and are recorded on a digital road map, link position represents the positions of all points on the link, link direction angles are the angle between the link and eastward direction, and link candidates are candidate points on the links nearest the car's estimated position.
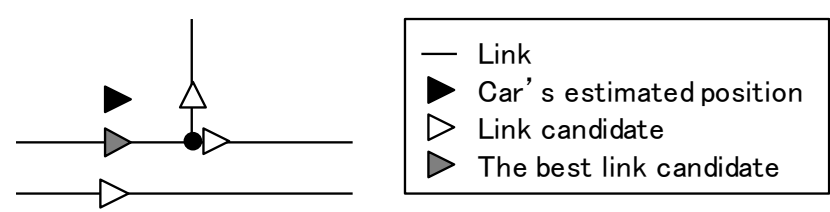

Fig. 1: Link, car's estimated position and link candidate

Many map-matching methods have been proposed and various criteria have been used to select the best link candidate [3]-[6]. As examples, the criteria are calculated from the positions of a car and a link candidate (Yang et al. 2003) [3], from the positions and the heading angle of a car and a link candidate (Kim et al. 2001) [4], from the accumulative distance and accumulative angle between a car and a link candidate (Morohoshi et al. 2008) [5], and from the number of visible satellites, the link facility code, the position and heading angle of a car, their error variances, and the positions and heading angles of link candidates (Pyo et al. 2001) [6]. There are drawbacks to these methods, however. Yang's method can match the car's estimated position to an incorrect link near an intersection when the positions of a car and a link candidate contain errors, because this method does not use the heading angles of a car and a link candidate. Kim's and Morohoshi's methods do not use the error variances of the positions, heading angle, accumulative distance and accumulative angle. Consequently, when the error variances increase, large errors occur in the criteria and the methods select an incorrect link candidate. Since Pyo's method do not uses the error variances of link position and direction angle, the error in the link position and direction angle sometimes causes the car's estimated position to be mismatched to a link on forks in roads, parallel road and so on.

In light of these drawbacks, the proposed method calculates the chi-squared statistic from the distance-series data of the positions, heading angles, and their error variances of the car and the link candidates. The term "distance-series data" refers to our usage of "a distance series" to describe a sequence of data points that are observed at constant distance intervals. Using the error variances of their positions and heading angles decreases the adverse influence on the criterion (the chi-squared statistic) even 
when the errors in their position and heading angle increase on forks in roads, parallel roads and so on.

This paper is organized as follows. Section 2 describes the structure of the car position measurement and the digital road map. Section 3 presents the proposed map-matching method that uses the chi-squared statistic. The results of experimental evaluation are presented in Section 4. Section 5 concludes the paper with a brief summary of key points.

\section{Structure of Car Position Measurement}

The car position measurement shown in Fig. 2 operates as follows. A GPS receiver outputs the car's position, speed, heading angle, the number of GPS signals being received and so on. The sensors are an odometer, a gyro sensor, an accelerometer and so on. They output the car's speed, angular velocity and acceleration. Position estimation observes the outputs of the GPS receiver and sensors and calculates the car's estimated position, speed, and heading angle and their error covariance, using a Kalman filter. Even though the system does not have any sensors, the car's estimated position, speed, heading angle and their covariance can be calculated from only the output of the GPS receiver. Map matching searches links near the car's estimated position, calculates criteria of link candidates from the outputs of position estimation and the data in the digital road map and selects the best link candidate. It determines whether the car is running on or outside a road [7]. If it is running on a road, the car-navigation system displays the position of the best link candidate. If it is running outside a road, the system displays the car's estimated position. We have proposed the road-departure-detection method [7]. This paper proposes selection of the best link candidate, which are the main processes in map matching.

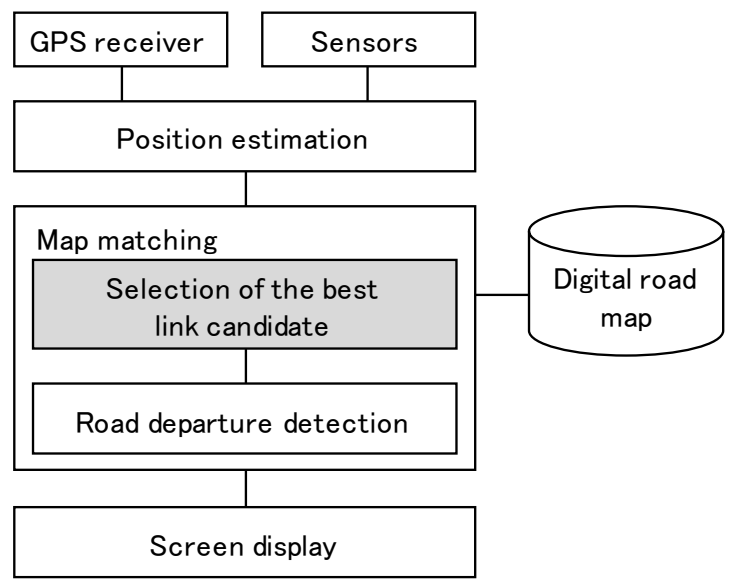

Fig. 2: Structure of car position measurement

The digital road map contains the information about roads, which are represented by links and nodes. Nodes and links respectively represent intersections and roads between intersections. Shape points are points on the link between the nodes. As shown in Fig. 3, the lines connecting the nodes and the shape points represent the road shape.
The attributes of a link are road type, number of lanes, road width, traffic direction, speed limit, and so on. The traffic direction are two-way traffic, one-way traffic and prohibited traffic [8], [9].
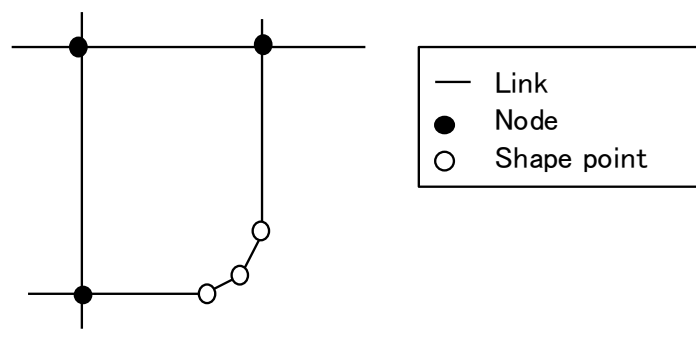

Fig. 3: Road network in digital road map

\section{Map-matching Method}

The following two conditions are satisfied when a car is traveling on a route.

Condition 1: The car's estimated positions and link positions do not differ.

Condition 2: The car's estimated heading angles and link direction angles do not differ.

We consider a criterion for indicating that Conditions 1 and 2 are satisfied. If the criterion is calculated from only the distance between the car's estimated position and the link position and the difference between the car's estimated heading angle and the link direction angle, a large error in the car's estimation or link causes map matching to select an incorrect link candidate. Therefore, the criterion is calculated from the normalized distance between the car's estimated position and the link position and the normalized difference between the car's estimated heading angle and the link direction angle. It is also difficult for map matching to select the best link candidate in parallel roads. However, because the shapes of roads to which parallel roads are connected differ, map matching can correctly select the best link candidate by calculating the criteria of the link candidates from the data of the past route (the distance-series data). It is assumed that errors in the car's estimated position and heading angle and those in the link position and direction angle are independently and normally distributed, where the error in the car's estimated position represents $\sqrt{e_{x e}^{2}+e_{y e}^{2}},\left(e_{x e}, e_{y e}\right)$ is the error in the car's estimated position in eastward and northward directions, the error in the link position represents $\sqrt{e_{x l}^{2}+e_{y l}^{2}}$, and $\left(e_{x l}, e_{y l}\right)$ is the error in the link position in eastward and northward directions. We consider the following criterion $T$ :

$$
T=\sum_{i=k-n+1}^{k}\left[\begin{array}{lll}
\Delta x(i) & \Delta y(i) & \Delta \theta(i)
\end{array}\right] \boldsymbol{\Sigma}_{d}^{-1}(i)\left\lfloor\begin{array}{c}
\Delta x(i) \\
\Delta y(i) \\
\Delta \theta(i)
\end{array}\right\rfloor
$$




$$
\begin{gathered}
\left\lfloor\begin{array}{c}
\Delta x(i) \\
\Delta y(i)
\end{array}\right\rfloor=\left\lfloor\begin{array}{l}
x_{e}(i) \\
y_{e}(i)
\end{array}\right\rfloor-\left\lfloor\begin{array}{l}
x_{l r}(i) \\
y_{l r}(i)
\end{array}\right\rfloor \\
\Delta \theta(i)=\theta_{e}(i)-\theta_{l}(i) \\
\boldsymbol{\Sigma}_{d}(i)=\boldsymbol{\Sigma}_{e}(i)+\boldsymbol{\Sigma}_{l}(i) \\
\boldsymbol{\Sigma}_{l}(i)=\left[\begin{array}{ccc}
\sigma_{p l}^{2} \sin ^{2} \theta_{l}(i) & 0 & 0 \\
0 & \sigma_{p l}^{2} \cos ^{2} \theta_{l}(i) & 0 \\
0 & 0 & \sigma_{\theta l}^{2}
\end{array}\right\rfloor
\end{gathered}
$$

where $\left(x_{e}, y_{e}\right), \theta_{e}$, and $\boldsymbol{\Sigma}_{e}$ are respectively the car's estimated position, heading angle and their error covariance matrix, $\left(x_{l r}, y_{l r}\right)$ is a position of the point near the car's estimated position in the running region (which, as shown in Fig. 4, is an area where a car can run), $\boldsymbol{\Sigma}_{l}$ is the error covariance matrix of the link candidate position and heading angle, $\sigma_{p l}^{2}$ is the error variance of the link position, $\theta_{l}$ and $\sigma_{\theta l}^{2}$ are respectively the link direction angle and its error variance, $i$ is the number of distance-series data elements, and $n$ is the number of distance-series data elements from which the criterion is calculated.

In Fig. 4, the origin of the coordinate system $(x, y)$ is an initial estimated position, and the positive directions of the $\mathrm{x}$-axis and $\mathrm{y}$-axis are respectively eastward and northward. The white circles are points near the car's estimated position in the running region, the black circle is a node or a shape point, the black line is the link, the black triangle is the car's estimated position, and the white triangle is the link candidate, which is a point near the car's estimated position on the link.

The distance between the car's estimated position and the link candidate position is calculated as follows. The car's estimated position and its covariance are obtained from position estimation. The running region is the area between the centers of the both side edges of lanes and between two lines that are perpendicular to the link and pass through the shape points or nodes. The running region is set by using the positions of the nodes and the shape points, the number of lanes, the road width and the traffic direction that are recorded in the digital road map. The point near the car's estimated position in the running region is calculated. Then the distance between this point and the car's estimated position is calculated. This distance is the distance between the car's estimated position and the link candidate position. As shown in Fig. 4, in the case of two-way traffic, the number of lanes in the running region is half of the number of lanes in the road. In the case of one-way traffic, the number of lanes in the running region is the same as that in the road. In the case of prohibited traffic, the running region and the link candidate are not set.

The link direction is a direction parallel to the link. The link candidate heading angle is the same as the link direction angle. The car's estimated heading angle, the link candidate heading angle and the link direction angle are all angles from the $\mathrm{x}$-axis.

The error covariance of the link candidate position is presented by Eq. (5) because the error in the link candidate position distributes in a direction perpendicular to the link. The error variances of the link position and direction angle are calculated beforehand. The procedure for calculating the error variance of the link position and direction angle is described in Paragraph 4.2.

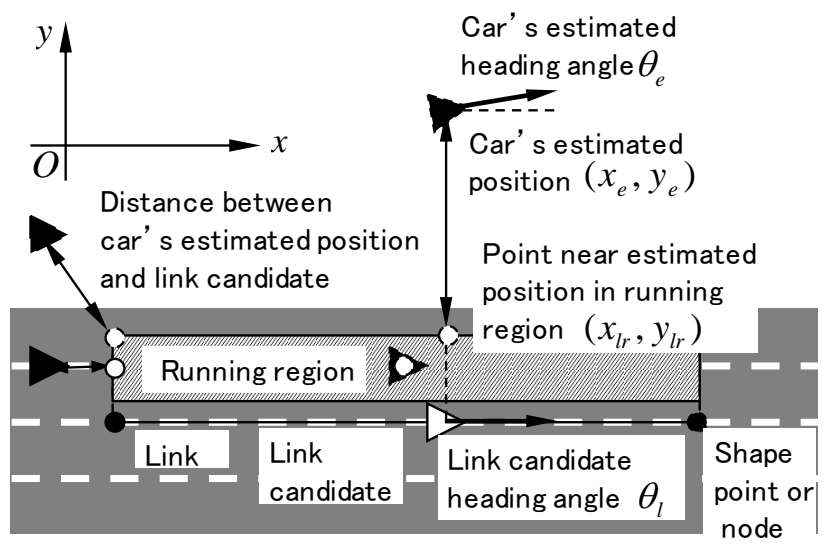

Fig. 4: Distance between car's estimated position and link candidate position

The criterion $T$ indicates the sum of the distance between the car's estimated position and the link candidate position and the difference between the car's estimated heading angle and the link candidate heading angle. Since the errors in the car's estimated position and heading angle and those in the link position and direction angle are assumed to be independently and normally distributed, where the error in the car's estimated position represents $\sqrt{e_{x e}^{2}+e_{y e}^{2}}$ and the error in the link position represents $\sqrt{e_{x l}^{2}+e_{y l}^{2}}$, it is chi-squared distributed with $2 n$ degrees of freedom. Therefore, the probability that the car is on a road is the value of the integral of the probability density function of a chi-squared distribution from the value of the criterion $T$ to infinity (Fig. 5). Fig. 5 shows the probability that the car is traveling on a route when the total number of distance-series data elements from which the criterion is calculated $(n)$ is 100 .

In the case that it is assumed that errors in the car's estimated position and heading angle and those in the link position and direction angle are independently and normally distributed, where the error in the car's estimated position represents $\left(e_{x e}, e_{y e}\right)$ and the error in the link position represents $\left(e_{x l}, e_{y l}\right)$, the criteria $T$ is chi-squared distributed with $3 n$ degrees of freedom. 


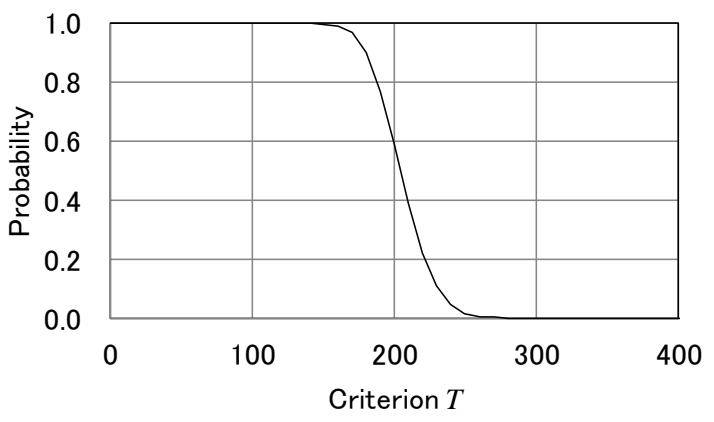

Fig. 5: Probability that the car is traveling on a route

Map-matching algorithm is shown in Fig. 6.

Step 1: Obtain the car's estimated position, heading angle and their variances from position estimation. Load link and node data from the digital road map. Calculate the positions of candidate points (link candidates) on the links nearest the car's estimated position and the distances between the links and the car's estimated position. Select the links which are located within a certain distance from the car's estimated position.

Step 2: Calculate the criteria of link candidates, using Eqs. (1)-(5).

Step 3: Select the best link candidate whose criterion is the smallest.

Step 4: Detect whether the car is running outside a road, using road-departure-detection method [7].

\begin{tabular}{|c|}
\hline step 1 \\
\hline Step 2 \\
\hline \multicolumn{2}{|c|}{ Calculate criteria of link candidates } \\
\hline step 3 \\
\hline step $4 \quad$ Select the best link candidate \\
\hline
\end{tabular}

Fig. 6: Map-matching algorithm

\section{Performance Evaluation}

\subsection{Data Collection Experiments}

For collecting observation data, a GPS receiver, an odometer, a gyro, and an accelerometer were installed in a car. The accelerometer measured acceleration in the up-and-down direction in the car. The gyro measured angular velocity in the revolution direction. The outputs of the GPS receiver and the sensors were recorded in a memory device at 1 and $10 \mathrm{~Hz}$.

The car run in environs and urban areas of Detroit, in urban areas of Paris, and in environs and urban areas of Moscow, and collected the output of the GPS receiver and the sensors. Table 1 shows places and times of data collection. The HDOPs (horizontal dilution of precision) and the numbers of GPS signals being received in Detroit, Paris and Moscow are shown in Figs. 7-12.

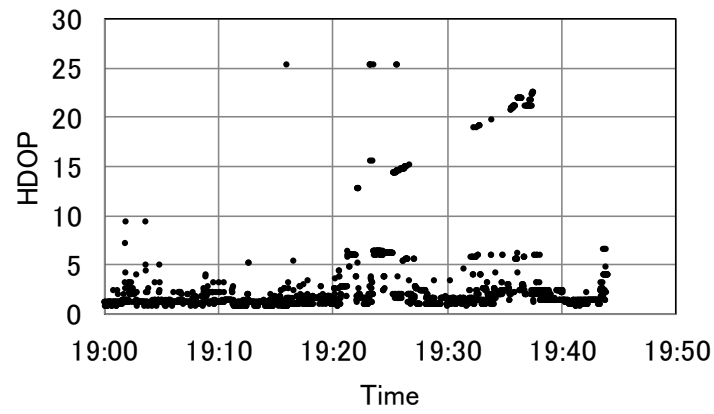

Fig. 7: HDOP in Detroit

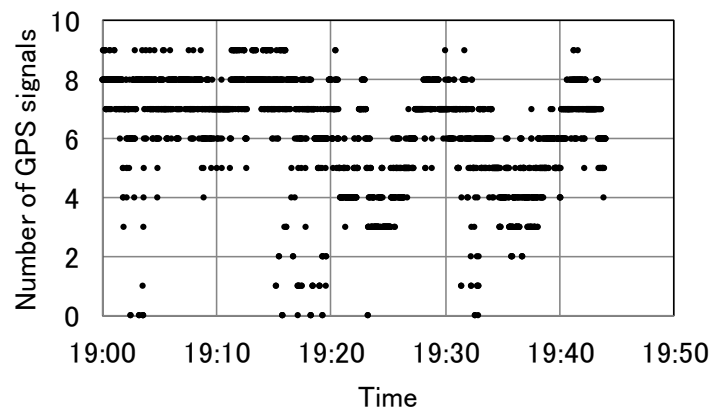

Fig. 8: Number of GPS signals being received in Detroit

Table 1: Places and times of data collection

\begin{tabular}{|c|c|c|c|}
\hline City, Country & Detroit, U.S.A. & Paris, France & Moscow, Russia \\
\hline Area type & Environs, urban area & Urban area & Environs, urban area \\
\hline Location & $\begin{array}{l}\text { around latitude } 42 \text { degrees } \\
20 \text { minutes north } \\
\text { and around longitude } 83 \\
\text { degrees } 2 \text { minutes west }\end{array}$ & $\begin{array}{l}\text { around latitude } 48 \text { degrees } \\
17 \text { minutes north } \\
\text { and around longitude } 2 \text { de- } \\
\text { grees } 51 \text { minutes east }\end{array}$ & $\begin{array}{c}\text { around latitude } 55 \text { degrees } \\
46 \text { minutes north } \\
\text { and around longitude } 37 \\
\text { degrees } 36 \text { minutes east }\end{array}$ \\
\hline Time and date & $\begin{array}{c}\text { 19:00:00-19:44:00 } \\
\text { September 11, } 2008\end{array}$ & $\begin{array}{c}14: 35: 18-15: 17: 51 \\
\text { March 27, } 2009\end{array}$ & $\begin{array}{l}20: 29: 34-21: 21: 14 \\
\text { February } 26,2009\end{array}$ \\
\hline
\end{tabular}




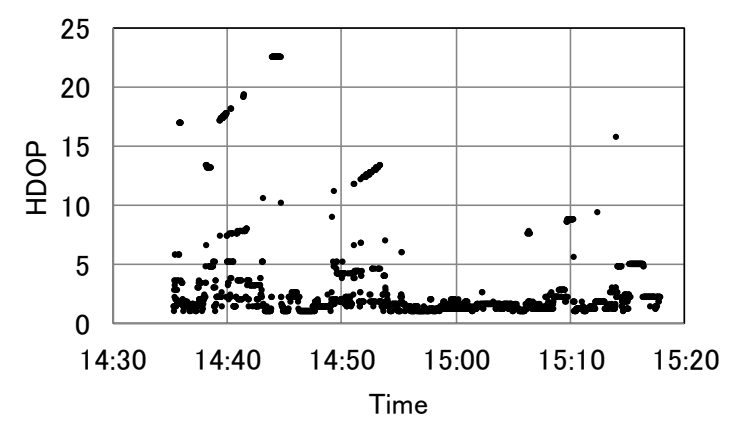

Fig. 9: HDOP in Paris

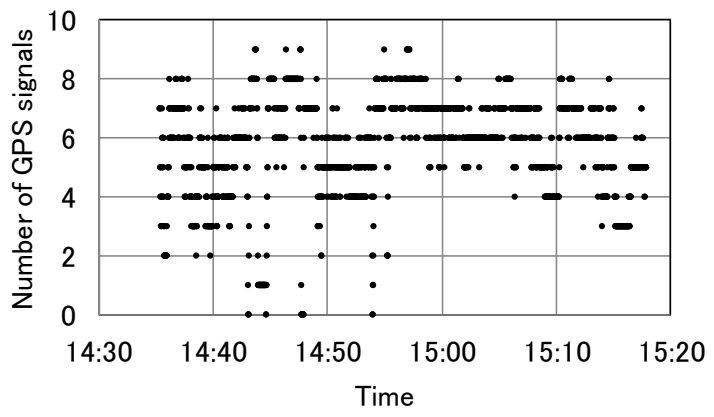

Fig. 10: Number of GPS signals being received in Paris

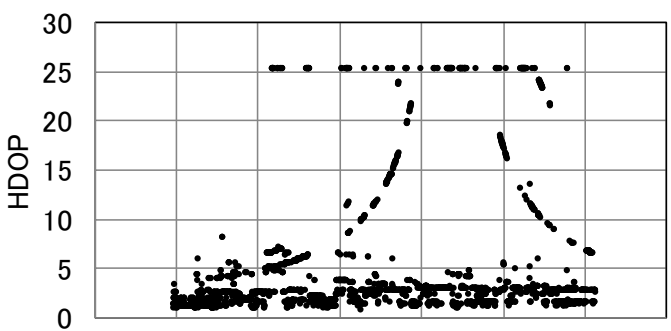

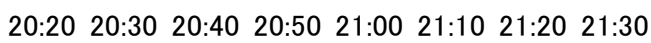

Time

Fig. 11: HDOP in Moscow

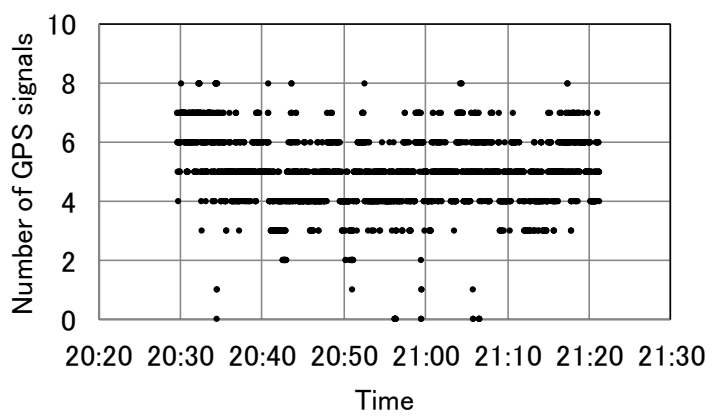

\subsection{Performance Evaluation Method}

In the evaluation, we first plotted links on aerial photo images, and measured the errors in link position and direction angle. The standard deviations of the errors in link position and direction angle ( $\sigma_{p l}$ and $\sigma_{\theta l}$ ) were calculated from the measured errors. We used a personal computer to process position estimation and map matching described in Section 3. Position estimation estimated the car's position, speed, and heading angle, and their estimation error covariance from the outputs of the GPS receiver and the sensors at 0.1-second intervals [1], [2]. Map matching was processed at 10-meter intervals, and the error covariance of the car's estimated position and heading angle $\left(\boldsymbol{\Sigma}_{e p}\right)$ were calculated by position estimation. The width of the running region (see Fig. 4) was calculated from the number of lanes, the road width and the traffic direction recorded in a digital road map.

It is difficult for map matching to correctly select the best link candidate when the car runs on forks in roads, parallel roads and roundabouts. We evaluated the map-matching performance of the method under these three situations using the collection datum. The evaluation indexes are correct map-matching rates. The correct map-matching rate in the case of forks in roads is calculated as the number of forks in roads on which the best link candidate is correctly selected when distance between two roads is within 10 meters divided by the number of all forks in roads on which the car ran. The correct map-matching rate in the case of parallel roads, and roundabouts which are calculated as the number of map-matching processes in which the best link candidate is correctly selected divided by the number of all map-matching processes.

\subsection{Performance Evaluation Results}

The correct map-matching rates in forks in roads, parallel roads and roundabouts are listed on Table 2. Even though it is difficult for map matching to correctly select the best link candidate, the correct map-matching rates in forks in roads and parallel were about $90 \%$, and that in roundabouts was more than $99 \%$.

Fig. 12: Number of GPS signals being received in Moscow

Table 2: Correct map-matching rate

\begin{tabular}{|l|c|c|c|}
\hline Road shape & Forks in roads & Parallel roads & Roundabouts \\
\hline Correct map-matching rate & $89.5 \%(35 / 39)$ & $91.6 \%(1003 / 1095)$ & $99.4 \%(167 / 168)$ \\
\hline
\end{tabular}




\subsection{Comparison with Traditional Method}

Traditional map-matching methods, for example Yang's method, Morohashi's method and Pyo's method, do not use the errors in the link positions and direction angles. Therefore, we compared with the traditional method which calculated the criterion $T_{t r}$ :

$$
T_{t r}=\sum_{i=k-n+1}^{k}\left[\begin{array}{lll}
\Delta x(i) & \Delta y(i) & \Delta \theta(i)
\end{array}\right] \mathbf{\Sigma}_{e}^{-1}(i)\left\lfloor\begin{array}{c}
\Delta x(i) \\
\Delta y(i) \\
\Delta \theta(i)
\end{array}\right\rfloor .
$$

This traditional method calculates the criterion without the error variance in the link positions and direction angles.

Almost links of forks in roads have errors in link positions and direction angles as shown in Fig. 13. Some links of parallel roads has errors in link positions and direction angles as shown in Fig. 14. We considered the conditions that the links of the road on which the car is running has the error, other links do not have the error, the errors in the car's estimated position and heading angle are zero meters and zero degrees respectively, and the value of other parameters are shown Table 3 . We compared map-matching performance between the traditional and proposed methods, processing the map-matching algorithm shown in Fig. 6.

In the case shown in Fig. 13 that the error in the link direction angle is 6.8 degrees on the forks in roads where the angle between roads is 12.4 degrees, the traditional method selects the incorrect link candidate, but the proposed method selects the correct link candidate. In the case shown in Fig. 14 that the error in the link angle is 5 degrees, the traditional method selects the incorrect link candidate, but the proposed method selects the correct link candidate, too. Consequently, the performance of the proposed map-matching method is better than that of the traditional method in the case that links of forks in roads and parallel roads have errors in the link positions and direction angles.

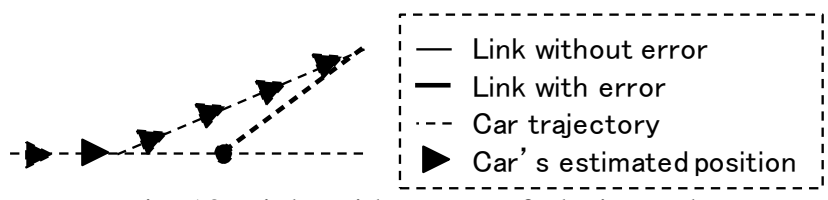

Fig. 13: Links with error on forks in roads

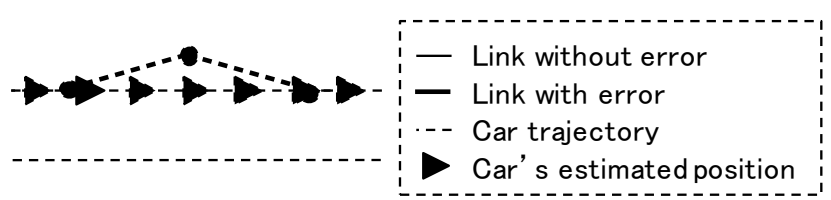

Fig. 14: Links with error on parallel roads
Table 3: Parameters for processing map matching

\begin{tabular}{|l|c|}
\hline \multicolumn{1}{|c|}{ Parameter } & value \\
\hline Error variance of car's estimated position & 9 meter $^{2}$ \\
\hline $\begin{array}{l}\text { Error variance of car's estimated heading } \\
\text { angle }\end{array}$ & 1 degree $^{2}$ \\
\hline Error variance of link direction angle & 25 degree $^{2}$ \\
\hline
\end{tabular}

\section{Conclusions}

We proposed a map-matching method that uses a chi-squared statistic for car position measurement. In selecting the best link candidate, it is able to take into account the error in the car's estimated position and heading angle, in the link position and direction angle because it calculates the selection criterion from their error variances. We experimentally verified that the method's efficacy on roads where it is difficult for map matching to correctly select the best link candidate. The correct map-matching rates were found to be about $90 \%$ in forks in roads and parallel roads, and more than $99 \%$ in roundabouts. We show that the performance of the proposed map-matching method is better than that of the traditional method in the case that links of forks in roads and parallel roads have errors in the link positions and direction angles.

\section{References}

[1] M. Bando, Y. Kawamata, and T. Aoki: Dynamic Sensor Bias Correction for Attitude Estimation using Unscented Kalman Filter in Autonomous Vehicle, Proceedings of the 42th ISCIE International Symposium on Stochastic Systems Theory and Its Applications, pp. 33-39, Okayama, November 2010.

[2] M. Bando, Y. Kawamata, T. Aoki, and K. Tanaka: The Study of Localization and Attitude Estimation for Autonomous Medium Speed Vehicle in an Outdoor Location, Proceedings of SICE 10th Annual Conference on Control Systems, 161-1-4, Kumamoto, March 2010.

[3] D. Yang, B. Cai, and Y. Yuan: An Improved Map-Matching Algorithm Used in Vehicle Navigation System, IEEE Proceeding on Intelligent Transportation Systems, pp. 1246-1250, 2003.

[4] S. Kim, and J. Kim: Adaptive Fuzzy-Network-Based C-Measure Map-Matching Algorithm for Car Navigation System, IEEE Transactions on Industrial Electronics, Vol. 48, No. 2, April 2001.

[5] H. Morohashi, K. Abe, Y. Tsukamoto, K. Uehara, and H. Seino: A Map Matching Algorithm using Vehicle Existence Coefficient, The 25th Sensing Forum, pp. 268-271, Saga, November 2008.

[6] J. Pyo, D. Shin, and T. Sung: Development of a Map Matching method using the multiple hypothesis technique, 2001 IEEE Intelligent Transportation Systems Conference Proceedings, pp. 23-27, Oakland, August 2001.

[7] T. Aoki, M. Bando, T. Hiruta, K. Kato, A. Kawabata, Z. Liu: Road Departure Detection Using State Dia- 
grams and Statistical Hypothesis Tests in Map Matching, Transactions of ISCIE, Vol. 26, No. 2, pp. 53-61, February 2013.

[8] K. Li, H. Tan, J. Misener and K. Hedrick: Digital Map as a Virtual Sensor - Dynamic Road Curve Re-construction for a Curve Speed Assistant, Vehicle System Dynamics, Vol. 46, No. 12, pp. 1141-1158, December 2008.

[9] J. Magenheim: Developing the AGORA road map ANDIL: AGORA Network against Digital Divide by means of Information Literacy, Joint Open and Working IFIP Conference ICT and Learning for the Net Generation, Kuala Lumpur, Malaysia, July 2008. 